\title{
Systems Engineering applied to the development of a Wave Energy
}

\section{Farm}

\author{
Diana Bull, Jesse Roberts, \& Robert Malins \\ Sandia National Laboratories, Albuquerque, USA \\ Jochem Weber and Kathrine Dykes \\ NREL, Golden, USA \\ Kim Neilson \\ Ramboll, Copenhagen, Denmark \\ Claudio Bittencourt \\ DNV GL, London, UK
}

\author{
Aurélien Babarit \\ LHEEA Lab., Ecole Centrale de Nantes - \\ CNRS, Nantes, France
}

Ronan Costello and Ben Kennedy

Wave Venture, Cornwall, UK

\begin{abstract}
This paper will introduce the Systems Engineering process utilized by the Structured Innovation team. Following the process this paper will present two key elements derived from the analysis of a successful Wave Energy Farm: the capabilities and the functions. The capabilities are the goals of a Wave Energy Farm as determined from a condensed list of the stakeholder needs. The functions are the activities or behaviors performed by the Wave Energy Farm in order to achieve the capabilities (i.e. the goals). At the framework level, both the functions and the capabilities remain independent of the design and these will be used to develop the list of requirements for a WEC in future work.
\end{abstract}

\section{MOTIVATION}

Wave energy converter (WEC) technology development as a whole has not yet delivered the desired commercial maturity nor, and more importantly, the techno-economic performance. Both commercial readiness and economic viability are required for successful entry into and survival in the worldwide electricity markets. The present day situation can be characterised with the following key points:

- Widely diverse WEC technologies are still being considered today

- No evidence of common convergence of technology implementation nor of underlying operational principles in key market segments (onshore, near-shore, off-shore)

- Techno-economic performance still requires considerable improvement for profitable economical application even if the expected cost reductions associated with economies of scale and learning curves are taken into account

- Current technology development has been rigid with respect to conceptual ideas, expensive, slow, and high risk with multiple technical and corporate failures coinciding with setbacks in prototype tests due to the focus on demonstration at large scale.

The ways in which WEC technologies have been and are being developed have been analyzed in more detail in [1] and [2].

Technology development progress, technology value, and technology funding have largely been associated with and driven by technology readiness, measured in Technology Readiness Levels (TRLs)
$[3,4]$. Originating primarily from the Space and Defense industries, the TRLs focus on procedural implementation of technology developments of large and complex engineering challenges where cost is neither mission critical nor a key design driver. Thus, appreciating lessons learned from WEC technology developments to date, it has become evident that there is a need for holistic, detailed and to the furthest extent possible, objective technology performance assessment at all stages of development.

The Technology Performance Level (TPL) [1-2] assessments can be applied at all technology development stages and associated TRLs. Even and particularly at low TRLs the TPL assessment is very effective as it considers a wide range of WEC attributes that define the techno-economic performance potential when developed to higher TRL; and highlights potential showstoppers at the earliest possible stage of the WEC technology development.

Systems Engineering (SE) is a disciplined approach to evaluating, holistically, the goals that must be achieved by a technology and the systems that enable achievement of the goals. This formal process which involves analyzing customer and stakeholder needs through the discipline of Systems Engineering offers a method to not only refine the TPL, but to also develop the requirements that will enable technical solutions that comprehensively address the needs of the stakeholders. These two outcomes are key goals of the Structured Innovation (SI) Project sponsored by the US DOE and led by NREL and SNL. The revised TPL assessment methodology and formulation of requirements will be used to identify 


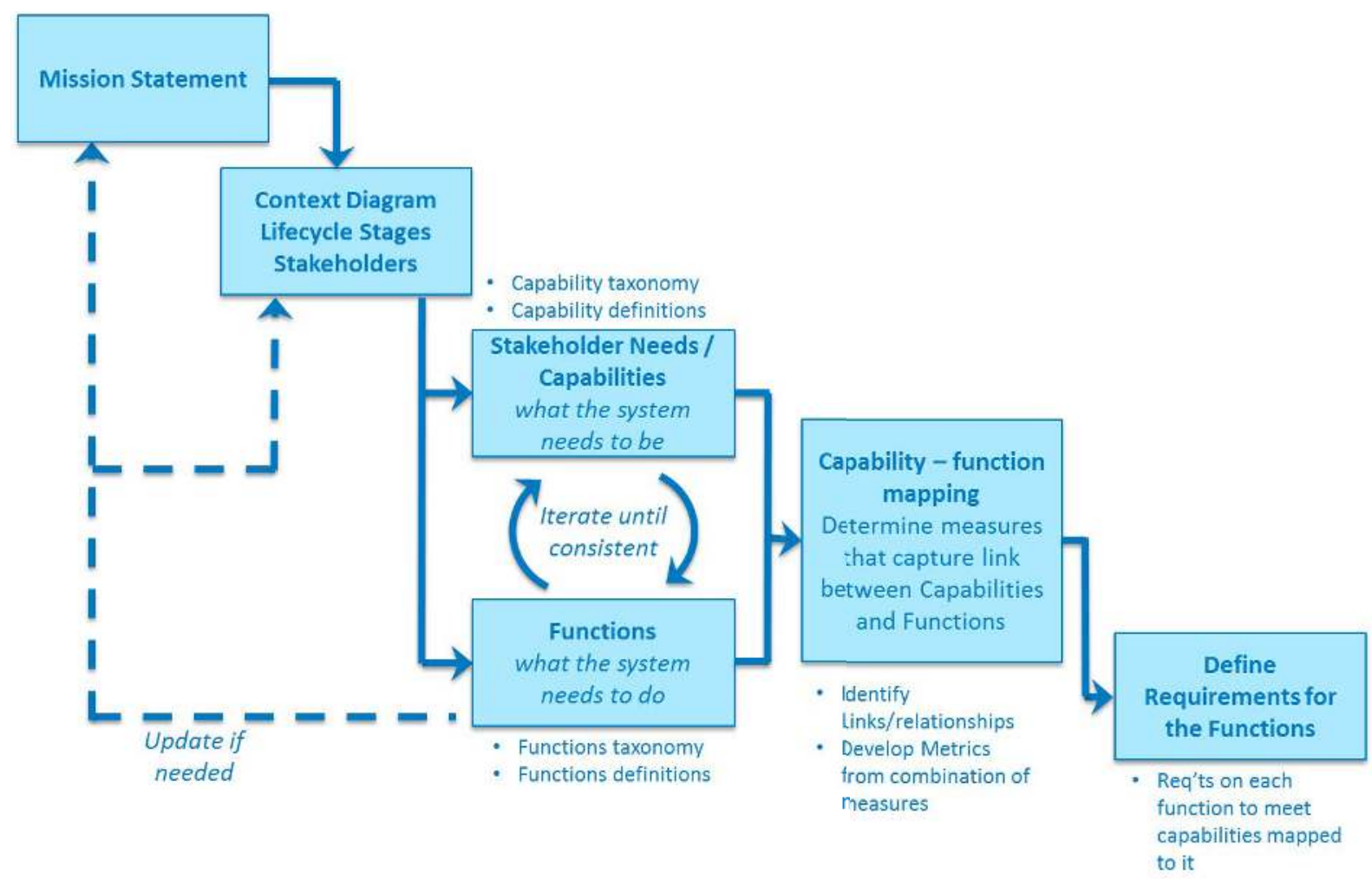

Figure 1: The systems engineering process applied to a Wave Energy Farm.

the necessary innovations that can yield high performing Wave Energy Farm (WEF) solutions.

\section{SYSTEMS ENGINEERING}

Systems Engineering is a rigorous application of processes and methods across a system's life cycle in order to ensure the adequacy of a system. The heart of systems engineering is a step-wise decomposition and flow down of stakeholder needs to each element of the system. The decomposition, flow down, and tracing of allocations ensures that the requirements and specifications for each subsystem, assembly, and component fully reflect and address stakeholder needs and adequately contribute to overall system performance. Systems engineering is most influential in the early stages when setting a mission and evaluating the stakeholders perspectives in the context of the environment in which the system will operate. The analysis outcomes are used first to create a functional architecture for the system and to decompose the stakeholder needs while allocating them to the system functions as requirements that are independent of the technology. The systems engineering work then continues through the development process to decompose the function-level requirements into specifications for each element of the physical design and to create the technical performance measures used to verify the design and validate the final system. The systems engineering process does continue through the rest of the system life cycle to provide the framework against which systems operations and maintenance are measured and candidate upgrades are evaluated. Numerous exam- ples of the success of the systems engineering approach can be found in the aerospace, defense, automotive, and oil and gas industries. This program has followed guidance from ISO 15288 [5], as well as IEE 1233 [6] with some tailoring according to the process described in ISO 15288 Annex A.

A wave energy farm is a complex system. The WEC, which itself is composed of multiple sub-subsystems like the power conversion, is just one subsystem in the farm. The mooring and anchoring, the point at which power is aggregated, the control centere and monitoring capabilities, and the delivery of electricity are all additional sub-systems. There are competing goals for each of these sub-systems and hence determining an optimal solution can only occur through a systems level understanding of the farm. Thus it makes sense to apply Systems Engineering to this problem. Surprisingly, there is no publically available reported work on the application of Systems Engineering to WEFs.

The systems engineering process used in this project is illustrated in Figure 1 and is a tailored version of the first steps of ISO 15288 (technical processes 6.4.1 to 6.4.4) [5]. The first activity was to develop a concise mission statement for the system (i.e., the WEF). This statement sets the framework for the development of the stakeholder needs and the functions. Capabilities and functions are hierarchical structures (i.e. taxonomies). In the case of capabilities, the taxonomy embodies the list of characteristics that are desired, from the perspective of the stakeholders, for the system to be successful. In terms of the functions, the hierarchy represents the 


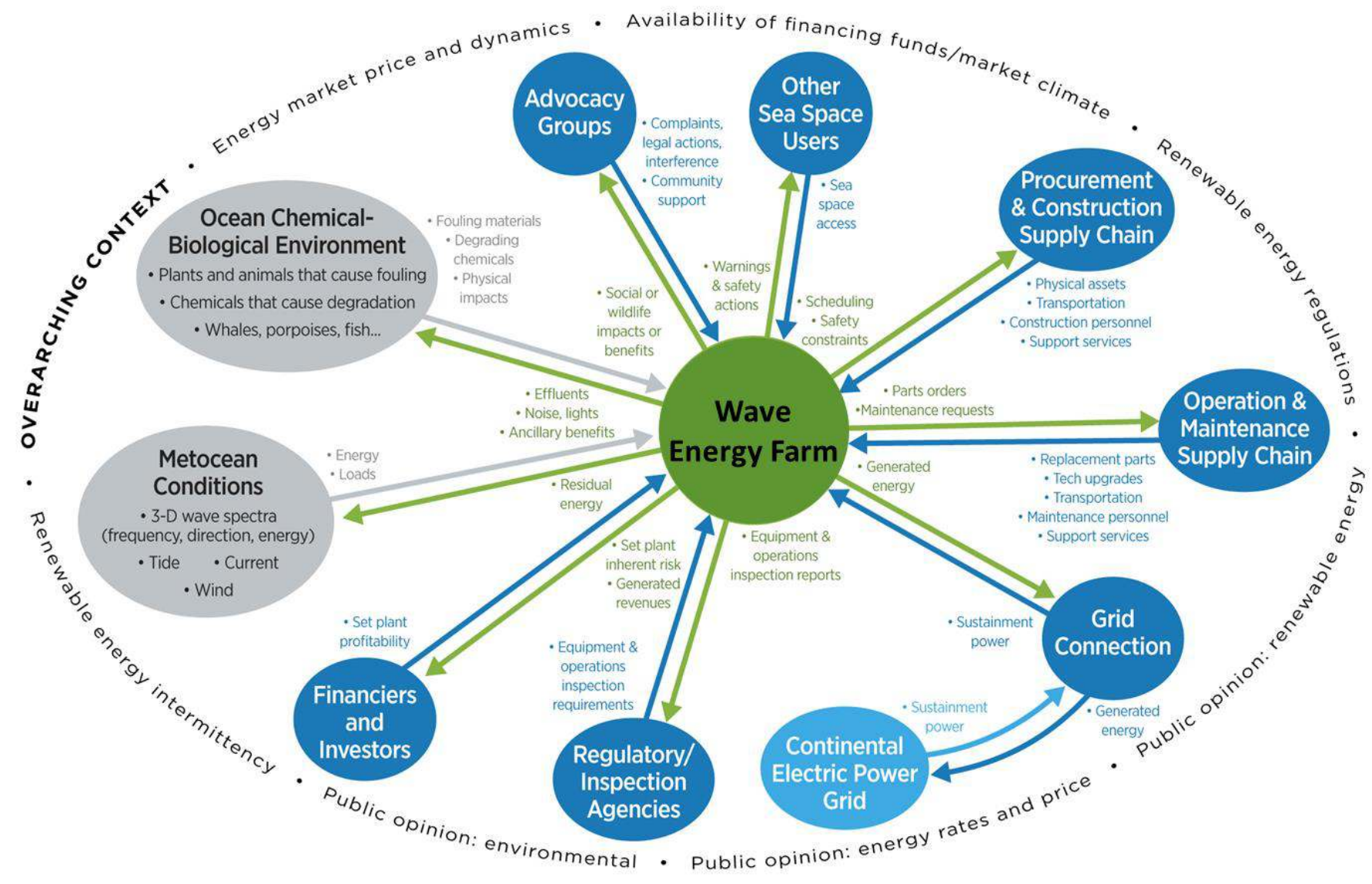

Figure 2: Context diagram for the Wave Energy Farm defining the problem boundaries and its environment.

solution agnostic (i.e. independent of specific design embodiments) elements that are needed to meet the stakeholder requirements. As indicated in Figure 1 this is an iterative process.

The entirety of the farm must be considered as the WEF dictates the success of the industry, not a subsystem within the farm. This project assumes a mature WEC technology industry with multiple competing certified WEC technologies that can achieve a cost competitive WEF. The requirements for a successful WEC unit will follow from the requirements for a successful WEF.

\subsection{Mission Statement}

The mission statement defines the criteria by which to measure a successful WEF. The entirety of the lifecycle must be considered within the mission statement. Six main lifecycle stages have been considered: Engineering, Procurement, Construction, Installation, Operations, and Disposal.

The systems engineering will consider the following as the mission of the WEF:

The wave energy farm will convert ocean wave energy to electricity and deliver it to the continental grid market in a competitive and acceptable manner across the lifecycle.

The precise language used in this mission statement allows for changing landscape whilst still specifying the application. Continental grid market means a very large grid market rather than any specialized niche market. While competitive sets a relative levelized cost of energy (LCoE) that will be driven by local market conditions (investors' appetite for risk, offering of feed-in tariffs, etc.). Competitive encompasses concepts of cost, availability, investor attractiveness, government incentives, and risk. Finally, acceptability incorporates the environment, regulation, insurability, safety, socio-economic, and social considerations.

\subsection{Context Diagram}

The context diagram is used to define the external systems that can directly influence the success of the WEF. In turn the operation of the WEF exerts an influence over these external systems. In some cases the external systems are stakeholders, but this is not necessary. Figure 2 shows the context diagram.

Surrounding the edge is a list of the overarching context in which the WEF operates. This list identifies the factors that are out of the control of the external systems and the WEF (political, social, and economic climate). However the overarching context can influence the external systems and the success of the WEF.

The WEF itself is composed of all the elements that are required to achieve the mission statement; further clarity on these elements can be found in Section 2.3. 


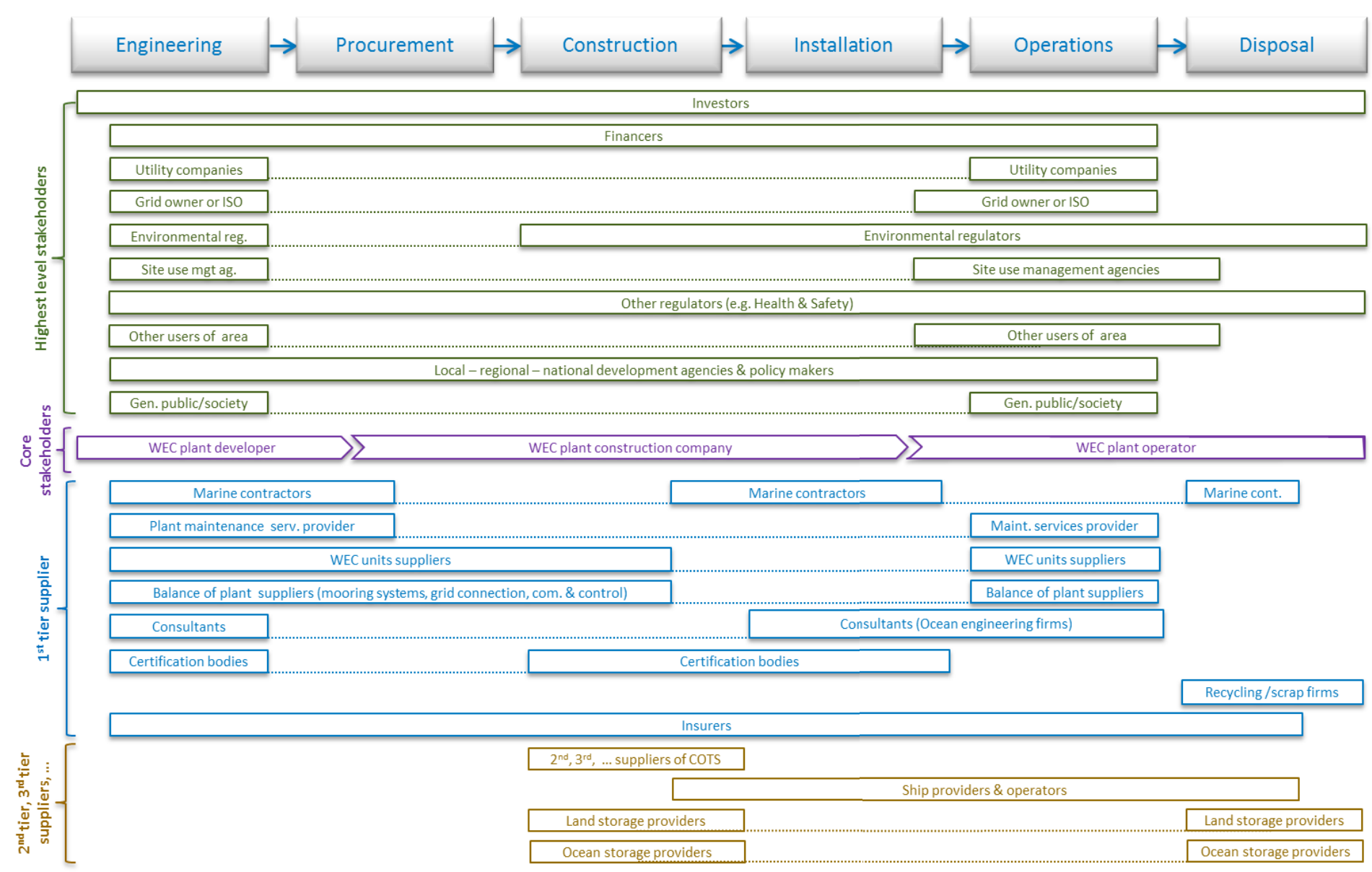

Figure 3: Identification of the 25 stakeholders, the lifecycle stage in which they are active, and the hierarchical tier they belong to.

\subsection{Stakeholders}

Using the lifecycle stages and the context diagram, all parties who would be involved in the WEF were identified. Figure 3 identifies the 25 stakeholders and at which lifecycle stage they are active. A total of 4 hierarchical groups are identified in Figure 3 through color. Each successive group of stakeholders puts constraints / requirements on the group below it. Hence this is a method of associating importance with a stakeholder whilst also identifying the specificity of their role. For instance, the investors will focus on the projected $\mathrm{LCoE}$, not on the requirements associated with the Marine Contractors. It is the project developer that will determine the requirements for the Marine Contractors.

\subsection{Capabilities}

The capabilities capture the stakeholder needs and desires in a distilled manner prior to selecting technologies or design approaches. The capabilities are independent of the solution or even the way in which a solution would be obtained.

Figure 4 identifies the capabilities that were derived by assessing all of the needs of all of the stakeholders. This condensed single list identifies what the system must be in order to ensure the WEF will achieve all the needs of all stakeholders. The top seven capabilities broadly identify the attributes of a successful WEF.

\section{C1: Have a market competitive cost of energy}

The electricity from the WEF may be sold on the day-ahead wholesale electricity market or through a Power Purchase Agreement (PPA). In both cases, the sale price of the electricity generated by the WEF shall be competitive with other energy sources. However, note that market price may vary among energy sources in some countries. There may be Feed-In Tariffs (FIT) for wave energy or renewable energy sources or there may be Renewable Energy Certificates or Renewable Obligations.

\section{C2: Provide a secure investment opportunity}

For investors and financiers, it is critical that WEF risks are well understood and manageable so that they know the financial risk, i.e. the risk that the farm will not deliver the expected financial return. The financial risk results from the analysis of the probabilities of the risks and of their financial consequences. Uncertainties on costs (CAPEX, OPEX) and revenues (energy production, availability, survivability) are the drivers. 


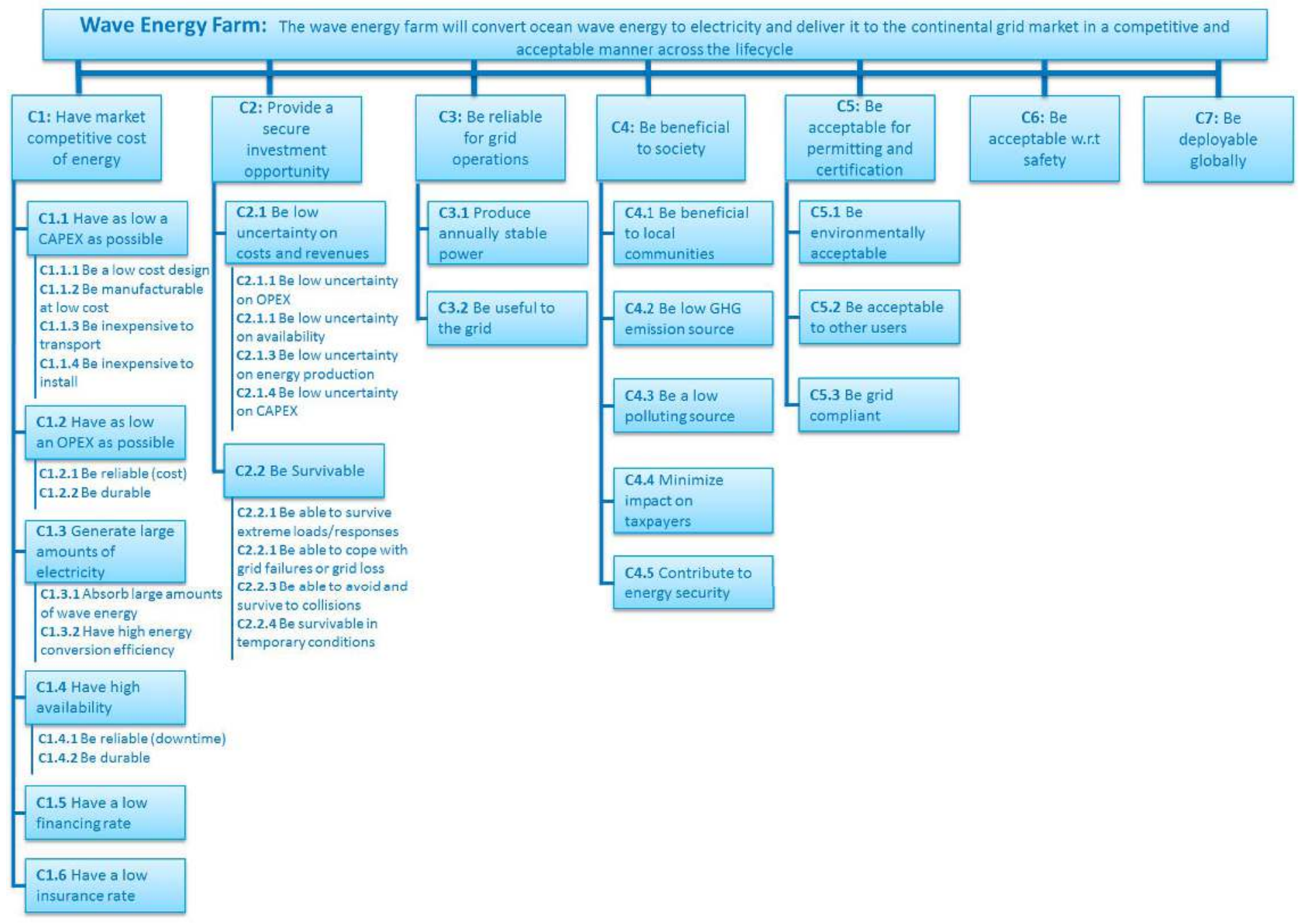

Figure 4: Taxonomy of the capabilities. This capability taxonomy identifies 33 distinct stakeholder needs.

\section{C3. Be reliable for grid operations}

Reliability for grid operations covers several aspects. The energy production from the WEF must be predictable sufficiently in advance to enter the day-ahead wholesale electricity market. Moreover, the increase of the share of intermittent renewable energy sources in the energy mix is challenging for grid operators with respect to (w.r.t) grid stability and load balancing. It could limit the deployment potential of wave energy. Thus, energy production from the WEF needs to be sufficiently consistent (short term variability) and the WEF needs to have a high capacity factor. Moreover, a WEF shall provide useful ancillary services to the grid. They include energy storage, automatic generation control (AGC), voltage and frequency control.

\section{C4: Be beneficial to society}

A WEF needs to obtain buy-in and support from the local communities and the general public. Indeed, as any industrial project, a WEF will have some negative impacts (higher cost of energy, disruption to other activities) that need to be largely overcome by benefits for society (low carbon emission energy source, local jobs creation, coastal protection). Otherwise, public con- cerns and actions against the project can seriously delay the project or make it fail (even if permits are granted).

\section{C5: Be acceptable for permitting and certifica- tion}

Permits for occupying the sea space and connecting to the grid must be obtained by the WEF developer before building the WEF (not having the permits is a critical risk for the farm developer, the investors and the financiers). Consequently, the WEF must fulfill all regulatory and permitting requirements. The requirements usually consist of assessing and addressing environmental impacts, impacts to other users of the area and impacts to the electrical grid.

\section{C6: Be acceptable with respect to safety}

Safety is a key requirement for any structure at sea. The WEF must be safe for manufacture, transport, construction, installation, commissioning, operations, and decommissioning in order to meet all other capabilities.

\section{C7: Be globally deployable}

The ability to provide steady sales is another key requirement for sustainable business for the WEF 
developer, construction company, and for the suppliers of the supply chain. It may also be an important requirement for the local-regionalnational development agencies, policy makers and general society w.r.t to the overall benefits. Thus, the WEF shall be deployable at many different sites, that represent a large global market share and be adaptable to variable site characteristics (wave resource, geophysical conditions, distance to shore, local infrastructure, ...). Global deployment is necessary for learning rates to take effect and to allow a WEF technical solution introduced to the market at TPL7 to ultimately reach TPL9.

The sub- and sub-sub-capabilities further specify the stakeholder needs that must be considered to either implement the capability or the sub-capability. For instance, the sub-capability ' $\mathrm{C} 1.1$ Have as low a CAPEX as possible' is achieved by: being a low cost design (C1.1.1), being manufacturable at a low cost (C1.1.2), being inexpensive to transport (C1.1.3), and being inexpensive to install (C1.1.4).

\subsection{Functions}

The functions define the fundamental elements of the solution that must be provided in order to achieve the mission and deliver the capabilities. High-level functions are independent of the technology or design used to implement the function. However, detailed functions may begin to border on specific design choices. Hence a strong effort has been made to maintain functions that are design agnostic.

Figure 5 identifies the technology agnostic functions that were determined as necessary to achieve the capabilities. The functions identify what the system must $d o$. Like the capabilities presented in Figure 4, the top level functions (6 of them) conceptually identify what the WEF must do to meet its mission.

\section{F1: Generate and deliver electricity from wave power}

The farm shall intercept the incoming hydrokinetic power in the ocean and convert it into electricity. The aim of this function is to generate as much energy as possible. It directly relates to the capability "be able to generate large amount of electricity from wave power".

\section{F2: Sustain Farm Operations / Maintain structural and operational integrity of WEF systems}

The farm shall support continual operations by controlling the position of the systems and by supplying energy. Further, the structural and operational integrity of the WEF and its systems' must be maintained throughout all environmental conditions, hence protection from the marine en- vironment and destructive responses shall be given. End stops and snap loads are typical challenges for this function. Its implementation may require buffers, end-stop springs or appropriate control strategies. PTO and electrical equipment may also require a controlled and vibration free environment. Position must be controlled to avoid collision between WEC units.

\section{F3. Provide integratable structural support}

The WEF system results of the integration of several physical sub-systems that result themselves of the integration of physical sub-sub-systems and components. The WEF shall integrate sub-systems and sub-sub-systems into a full and safe farm.

\section{F4: Control Farm Operations}

The farm must be capable of continued knowledge and support of its systems' and their states'. The farm operator shall be able to determine the appropriate control decisions for the farm and its constituent sub-systems. The necessary instrumentation, telemetry, processing, synthesis, and communication shall be present to inform the control actions and confirm successful implementation of control actions. In normal operations, the control may be implemented by an automated farm control system.

\section{F5: Enable safe operations}

The farm and its constituent sub-systems shall employ internal and external mechanisms and measures that achieve safe surroundings during all operational modes: installation, operation, and maintenance and repair. Particular attention should be applied to maintenance events when personnel must interact directly with the farm. These mechanisms and measures will meet or exceed the requirements specified in the obtained permits.

\section{F6: Provide synergistic benefits}

The farm can provide positive environmental, social, or grid services.

\section{CAPABILITY - FUNCTION MATRIX}

The capabilities and the functions are united; the functions identify what the system must $d o$ in order to achieve what be system must be, i.e. the capabilities. As such, there are measures that can be identified at the intersection of functions that actually impact or implement a given capability. These measures are concrete and they form both the basis of the drivers for the TPL assessment as well as the requirements for the farm. Figure 6 illustrates a generalized version of this concept. 


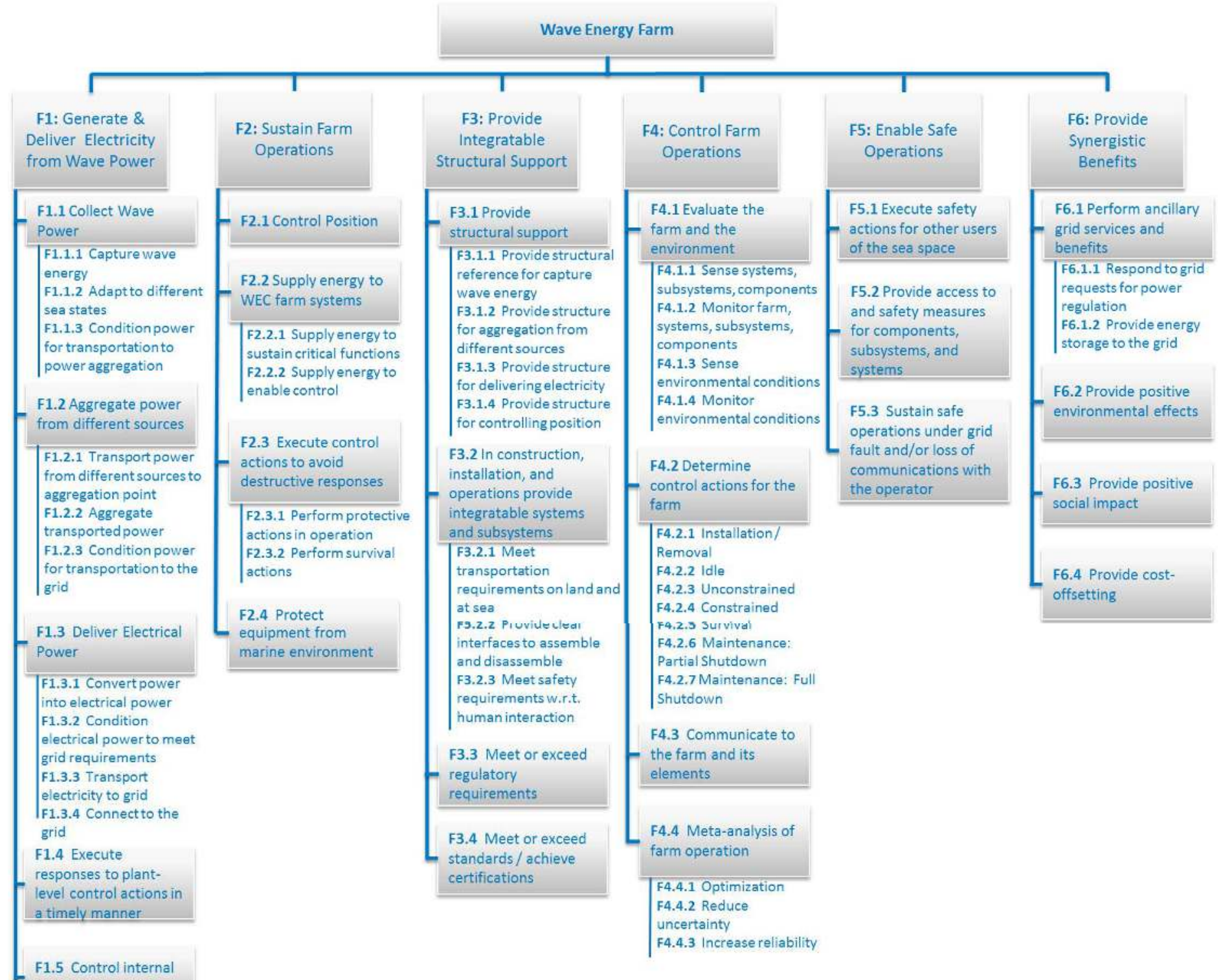

system processes.

Figure 5: Taxonomy of the functions. The function taxonomy identifies 67 elements needed to achieve the WEF mission.

\subsection{Technology Performance Levels (TPLs)}

The TPL is designed to be an assessment of the suitability of the technical solution for the customers' needs. The TPL groups and attributes, originally developed through experience [1], are now recognized as the capabilities. By compressing all of the measures that have been identified across all of the functions, i.e. by collapsing all columns into one, the measures by which to evaluate the capability can be determined and prioritized.

Tradeoffs in the overall design manifest themselves in the competing capabilities. The specific technical solutions chosen for a design are rated against the specific measures for each capability. This identifies which tradeoffs a technology has chosen. For instance, in order to be a low cost design a device should not require a lot of material. However, in order to be able to generate a large amount of electricity the device should be large. Hence as the TPL is assessed on a holistic level, if you choose to favor small amounts of material you will receive a high score for this assessment criteria, but it may be balanced by a low score in generation.

The application of this Systems Engineering approach to the TPLs is almost complete. It is expected that a revised version of the TPL assessment methodology will be publically released soon.

\subsection{Requirements}

The functions presented in Section 2.3 set the conceptual requirements, i.e. the farm must be able to generate and deliver electricity from wave power. Setting these conceptual expectations is necessary in order to develop the measures by which the functions can be evaluated. Hence, by compressing all of the measures that have been identified across all of the capabilities, i.e. by collapsing all rows into one, the farm requirements can be determined from analysis of all of the measures. These requirements are much more specific and may be matched with quantitative expectations. 


\begin{tabular}{|c|c|c|c|c|c|}
\hline \multirow{2}{*}{\multicolumn{2}{|c|}{$\begin{array}{l}\text { Capabilities - functions } \\
\text { mapping matrix }\end{array}$}} & \multicolumn{4}{|c|}{ Functions } \\
\hline & & \multirow{2}{*}{\begin{tabular}{|l|} 
Function 1 \\
Measure 1 \\
\end{tabular}} & \multirow{2}{*}{\begin{tabular}{|l|}
$\begin{array}{l}\text { Sub-function } \\
1.1\end{array}$ \\
\end{tabular}} & \multirow[t]{2}{*}{ Function 2} & \multirow[t]{2}{*}{ Function $\mathrm{F}$} \\
\hline \multirow{5}{*}{ 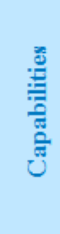 } & Capability 1 & & & & \\
\hline & Capability 2 & & & Measure 1 & Measure 1 \\
\hline & Sub-capability & & Measure 1 & & \\
\hline & 2.1 & & Measure 2 & & \\
\hline & Capability C & & & & Measure 1 \\
\hline
\end{tabular}

Figure 6: Generalized capabilities - functions mapping matrix.

The development of the requirements and the refinement of the functions is ongoing work within the program. Each requirement should possess the following properties:

a. Abstract. Each requirement should be implementation independent.

b. Unambiguous. Stated so that it can be interpreted in only one way.

c. Traceable. Each requirement should be traceable to a specific documented customer need.

d. Validatable. Need to check if a proposed/completed system satisfies the requirements.

\section{CONCLUSIONS}

This paper has presented the initial results obtained from the application of Systems Engineering to a Wave Energy Farm system. A mission statement has indicated that this work is interested in achieving a market-competitive and acceptable WEF. Twentyfive stakeholders were identified as active throughout the lifecycle of this WEF.

The stakeholder needs were condensed into a list of 33 capabilities - i.e. what the WEF must be in order to be successful for all of the stakeholders identified. In turn these capabilities will be the basis of the revised TPL assessment.

In conjunction with identifying what the WEF must be, what the WEF must do was also identified through 67 functions. These functions and the concrete measures that link the capabilities to the functions will be used to develop requirements for a successful WEF.

\section{ACKNOLEDGEMENTS}

This work is part of the Structured Innovation Project. This work was funded by the U.S. Department of Energy's Wind and Water Power Technologies Office. Sandia National Laboratories is a multiprogram laboratory managed and operated by Sandia Corporation, a wholly owned subsidiary of Lockheed Martin Corporation, for the U.S. Department of En- ergy's National Nuclear Security Administration under contract DE-AC04-94AL85000.

\section{REFERENCES}

[1] J.W. Weber, "WEC Technology Readiness and Performance Matrix - finding the best research technology development trajectory", in Proc. 4th International Conference of Ocean Energy, Dublin, Ireland, 2012

[2] J.W. Weber, R. Costello, J. Ringwood, 2013, "WEC Technology Performance Levels (TPLs) - metric for successful development of economic WEC technology", in Proc. Of the $10^{\text {th }}$ European Wave and Tidal Energy Conference (EWTEC2013), Aalborg, Denmark

[3] J.Fitzgerald et. al. "Technology Readiness for Wave Energy Projects; ESB and Vattenfall classification system”, In Proc. $4^{\text {th }}$ International Conference on Ocean Energy (ICOE2012), Dublin, Ireland, 2012.

[4] K. Ruehl and D. Bull, "Wave Energy Development Roadmap: Design to commercialization," in OCEANS '12. "Harnessing the Power of the Ocean". Proceedings, Hampton Roads, VA, 2012, pp. 1-10.

[5] International Organization for Standardization. ISO/IEC 15288:2008 Systems engineering -- System life cycle processes, 2008.

[6] IEEE 1233 “Guide for Developing System Requirements Specifications". Institute of Electrical and Electronics Engineers (http://www.standards.ieee.org/) 